\title{
THE ACHIEVEMENTS OF THE "COMPUTER INTEGRATED ROAD CONSTRUCTION" PROJECT
}

\author{
François Peyret
}

\author{
Site Robotics Subdivision, Laboratoire Central des Ponts et Chaussées \\ E-mail: francois.peyret@lcpc.fr
}

\begin{abstract}
The CIRC project has been the first major European project aiming at introducing the benefits of new technologies into the road work sites. After having introduced the project and its functional objectives, the paper describes the two products that were developed, the one for the compactors and the one for the asphalt pavers. A special stress is given to the major innovations that have resulted from the project. The paper end with the description of the achievements in terms of industrialisation of the products.
\end{abstract}

Keywords: computer integrated construction, $\mathrm{CAD}$, real-time positioning, work site

\section{INTRODUCTION}

The "Computer Integrated Road Construction" (CIRC) project, $\mathrm{n}^{\circ}$ BE-96-3039, has been supported by the European Commission, under the Industrial \& Materials Technologies Programme Brite-EuRam III. It aimed at demonstrating the interest of new tools, based upon the application of state-of-the-art information technologies to the road construction sites.

Seven partners were involved in the project, each providing complementary skills and abilities (10) :

- ITMI division of CAP GEMINI France,

- the Laboratoire Central des Ponts et Chaussées (LCPC), France,

- TEKLA OY, Finland,

- University of Karlsruhe, Germany,

- University of East London (UEL), Great Britain,

- EUROVIA, France,

- National Land Survey (NLS), Sweden.

The consortium has developed during the project two different versions of CIRC systems for the first two target machines : CIRCOM for compactors and CIRPAV for pavers.

\section{OBJECTIVES}

\subsection{General stakes of the CIRC project}

The paradigm of computer integrated construction (CIC) means the sharing of a common numerical geometrical data base from the design, through all the site operations up to the quality control of the geometry of the structure [1] [9]. It relies upon the main following technologies: Computer Aided Design (CAD), automatic control, short-range wireless communication and particularly, the key technology that was the latest to be developed: real-time positioning.

The CIRC project aimed to develop CIC systems for road construction equipment, to be integrated, in a first step, into compactors and asphalt pavers.

\subsection{Functional objectives of CIRCOM}

In road layers compaction, it is essential that the right level of energy should be transmitted to the material, with a uniform distribution. This energy, as far as the settings of the compactor do not vary, depends directly from the number of runs, or passes, of the machine [4] [5].

So, the first and main objective of CIRCOM was to assist the driver in this task, so that he can perform the exact number of passes, at the right speed, everywhere on the surface to be compacted. To perform this task, it is essential to provide the system with an accurate and continuous localisation of the machine.

As, generally, 2 to 4 compactors work together on big sites, CIRCOM, in its multi-compactors version, has to manage the data base globally for all the fleet.

The second objective of CIRCOM was to record the actual work achieved by the roller, in terms of trajectory followed and number of passes achieved on every point of the trajectory, to feed the site data base and perform a global quality control at the site level.

\subsection{Functional objectives of CIRPAV}

In Europe, most of the pavement courses are laid by pieces of equipment called "asphalt pavers" or "finishers". When it is used to spread the sub-base and base layers, it generally uses a reference in altitude, in order to recover the final project profile and to correct the generally big defects of the capping layer. This reference is generally materialised by string lines (also called wire ropes), installed along the road. These 
wires are expensive to install, dangerous for the workers and generate problems for site truck traffic [2]. More and more often, on big work sites, the string line is replaced by a plane generated by a rotating laser beam and the sensors are photo-cells. However, this device provides only a reference plane and is not really convenient for controlling the tool along an undulating profile, as most of the roads are. For these reasons, they are quite inappropriate to a real industrialisation of the sites.

So, the two main functions that have to be ensured by CIRPAV, are: the assistance of the driver in his task of maintaining the paver on its correct trajectory at the right speed and the assistance of the screed-man in his task of controlling accurately the position of the screed, either manually or automatically. For these two goals, CIRPAV must use data coming from a real positioning system, capable of determining accurately and totally the geometry of the laid course and to localise with reference to the project.

As for CIRCOM, the secondary objective concerning the recording of all the as-built data, for quality control, was maintained.

\section{BENEFITS OF CIRC PRODUCTS}

The main expected economic gains brought by CIRC products are at four levels:

- saving of tedious labour cost, in terms of setting out and manual control of the geometry (an analysis made by EUROVIA showed that a 80 kEuro CIRPAV could be paid off thanks to this saving on a single 30 $\mathrm{km}$ long highway work site),

- saving of equipment use, thanks to the fact that the trajectories are achieved much more accurately (it was calculated that two CIRCOM, of $60 \mathrm{kEuro}$ each, could be paid off by this saving on two $30 \mathrm{~km}$ long highway work sites),

- saving of material, thanks to the better altitude control, enabling smaller tolerances in the design with the same result in terms of mechanical resistance (around 3500 Euros / km on a 2 × 2 way highway pavement site),

- improvement of quality and consequently suppression of supplementary costs for repairing lowquality works.

\section{DELIVERABLES}

The main deliverables of the project have been the two CIRCOM and CIRPAV prototypes.

Each of them is broken down into three subsystems: ground sub-system, positioning sub-system and on-board sub-system (see Figure 1).

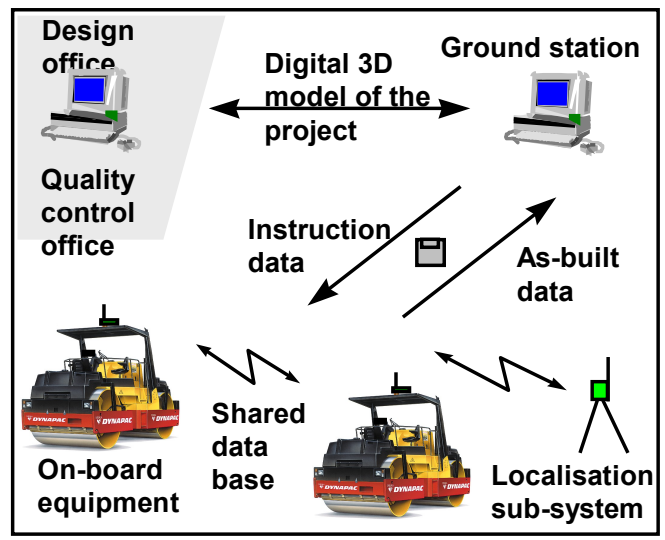

Figure 1: general architecture of a CIRC system

\subsection{CIRCOM prototype}

The prototype had already been described in [7] and [8].

\subsubsection{Ground sub-system}

The ground station is used at several stages of the road project:

- feeding of the database of the ground station with the project data,

- preparing every day the mission for each compactor of the fleet, using the user-friendly graphical interface,

- at the end of the day, updating the work-site database with the results of the day.

This task is performed by a specialised software running on a standard office $\mathrm{PC}$, which is used by the supervisor of the work-site. The core of this software package is common to CIRCOM and CIRPAV. The most innovative feature of it, not visible but fundamental, is the structure of the data base itself which is 3-dimensional, meaning that all the elements of the project, as well as the trajectories, are stored as $3 \mathrm{D}$ polylines in a vector data base.

\subsubsection{Positioning sub-system}

It had been chosen to use state-of-the-art Global Positioning System (GPS) technology, in real-time kinematic (RTK) mode, as main technology for its accuracy and its basic advantages of space-consistency (the 3 co-ordinates are intrinsically linked) and timeconsistency (measurements are perfectly repeatable, whatever the operator and the conditions).

However, to overcome the availability problems of the GPS signals, existing in zones where the satellites are not visible (particularly under the bridges), an integrated system has been designed, using deadreckoning sensors (Doppler radar, encoder and fibreoptical gyrometer) in addition to GPS. To get the best from all the sensors, an extended Kalman filter [8] has been designed, running at the rate of $25 \mathrm{~Hz}$. 


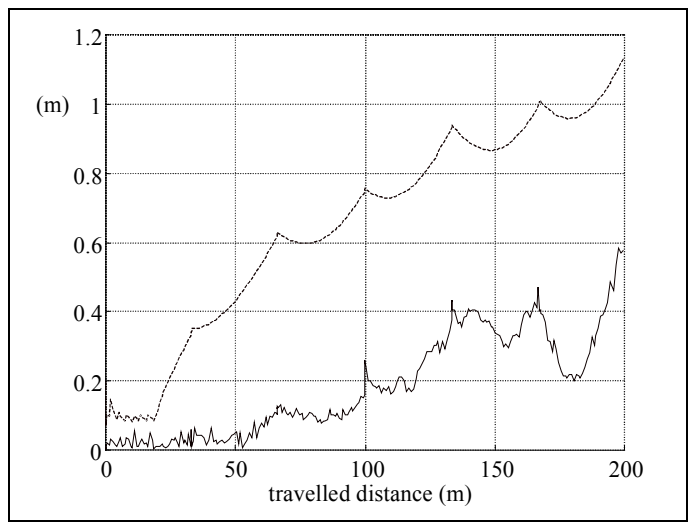

Figure 2: accuracy of CIRCOM positioning system in case of GPS shadowing

Figure 2 illustrates the performances of CIRCOM sub-system when the machines follows this trajectory. The lower curve in solid line represents the absolute error. One can check that it remains smaller than 20 $\mathrm{cm}$ during the first $100 \mathrm{~m}$, matching that way the required accuracy. Mostly all the results coming from full-scale tests are equivalent, even better.

\subsubsection{On-board sub-system}

The role of the on-board sub-system embedded on the compactor is to:

- manage a man-machine interface (MMI), which assists the driver in compacting, data,

- process in real time instruction data and position

- memorise work done (trajectories),

- exchange achieved work data with other compactors working on the same section.

These tasks are performed by robust, industrial PC equipped with high brightness colour LCD screen, PCMCIA removable media and reduced keyboard. The machine driver can visualise this way in real time his own position, his colleagues positions, the total number of passes realised upon the whole section by all the rollers, together with his actual speed compared to the reference speed. Figure 3 presents the manmachine interface (MMI) of CIRCOM.

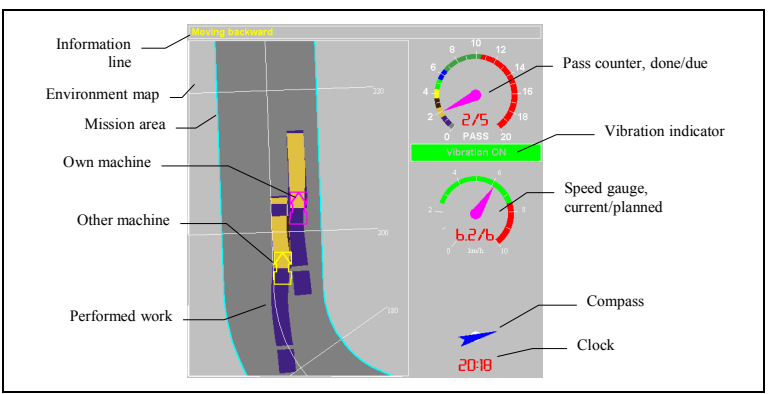

Figure 3: CIRCOM on-board sub-system MMI

The main innovations which have been developed in the frame of the project for the on-board sub-system are: an original vector data-base for road machine guidance and a set of new technological solutions allowing the multi-machines functionalities.
The road data-model was designed to satisfy several criteria in terms of universality, robustness, processing and displaying efficiency. The model is based upon concatenation of 3D quadrilaterals, socalled "ribbons", which are capable of representing any kind of road object useful to "intelligent" machines, using a CIC-type system, as it was introduced at the beginning of this article. Figure 4 shows some examples of ribbons, 2 "thin" ones describing road axis and road edge, and one "thick" one describing the trace of the roller.

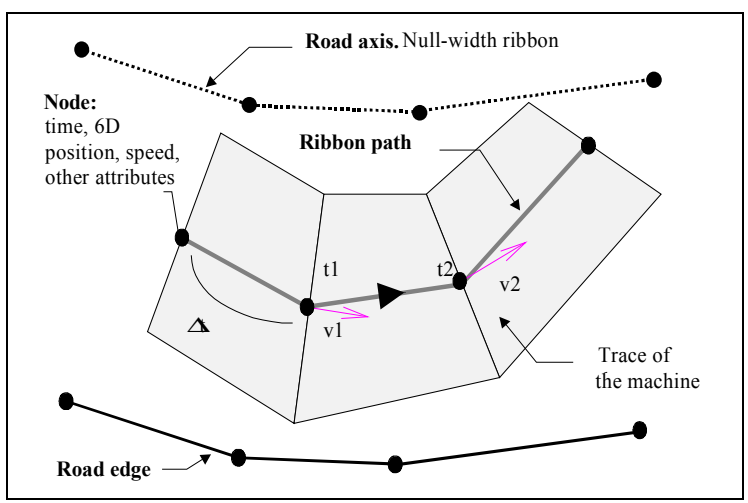

Figure 4: examples of 3D "ribbons"

The specific solutions to multi-CIRCOM are based upon: an architecture, a communication principle and a communication protocol, a communication hardware choice.

The chosen architecture, rather than "client-server" (also called "master-slave"), has been the "peer-topeer" one, meaning that all the stations are absolutely equivalent and possess the same amount of data.

The communication principle has been optimised taking into account the particular features of the application. Every compactor emits at regular intervals (of the order of $5 \mathrm{~s}$ ) the minimum amount of information required by the other machines to update their data-base. In case of missing information, a special request is sent and an optimisation algorithm determines who is the most appropriate colleague in the range capable of sending him the requires data. The machine entering the section after a long absence receives automatically all the missing information, but this one is transmitted with a low priority, in order not to saturate the current exchanges.

The chosen communication protocol has been TCP/IP, a commonly used standard for Internet communication, guaranteeing this way a perfect compatibility with emerging communication technologies, all supporting this standard.

From a technological point of view, 2 solutions have been studied and are compatible with multiCIRCOM: standard radio-modems, capable of communicate over distances of several kilometres, but with a relatively slow rate (10 to $20 \mathrm{kbits} / \mathrm{s})$ or "WaveLan" modules, developed to prolong office networks over distances not exceeding several hundred metres $(700$ to $800 \mathrm{~m})$, but with a quite high rate of at least $1 \mathrm{Mbits} / \mathrm{s}$. The latest solution has been chosen for 
the prototype and successfully demonstrated on an experimental work site.

\subsection{CIRPAV prototype}

\subsubsection{Ground sub-system}

As for CIRCOM, the role of the CIRPAV ground sub-system is to:

- provide the paver with geometric data about the work-site, coming from CAD data, as well as guidelines for operation,

- prepare the asphalt paving mission,

- compute paving results and prepare statistics on the work achieved.

This task is performed by a specialised software, which is an extension of the CIRCOM ground station software.

The user chooses a paver (and its associated parameters) and defines one or several trajectories. To create a trajectory, the user selects, on the map, a road polyline, and gives values for the start and end section, and start and end offset from the selected polyline.

In order to visualise the paving work results, there are three views the user can adopt: the map view, the cross section view and the profile view:

- the map view shows the differences between the achieved surface and planned surface. The differences between the two surfaces are shown with different fill colours,

- the cross section view shows the cross section of the target and achieved surface, at a selected section of the road,

- the profile view shows the profile along the road of the target surface and the achieved surface, with a selected offset from the axis. Profiles with different offsets can be projected to the profile view.

The user can also ask the system to produce statistical results, such as altimetry listings and paver' speed diagrams.

\subsubsection{Positioning sub-system}

The role of the positioning sub-system is to locate precisely and in real-time the paver and to get the exact geometrical description of the layer currently being spread. For that purpose, different sub-systems were designed, in order to provide the best possible solution, with regards to the specific constraints of the paving site.

Mainly two technologies were addressed:

- the RTK-GPS technology, as for CIRCOM, combined with inclinometer measurements, for paving works less demanding in terms of elevation accuracy,

- a novel 6D laser-based technology, capable of generating continuously and automatically sets of 6 spatial co-ordinates ("LaserGuide" system from University of East London [3], with an excellent accuracy in terms of elevation.

GPS solution

Clearly, this first solution should provide a more automated and industrial solution, thanks to the global aspect of GPS, which brings both space and time consistencies [9]. Moreover, it should bring a perfect compatibility with CIRCOM, enabling to use only one type of positioning technology on the site. However, the best accuracy in elevation that it is possible to achieve with a state-of-the-art RTK receiver, under usual site conditions, is $\pm 2 \mathrm{~cm}$ and this does not meet the requirements for most pavement layers. Furthermore, there are still availability problems with GPS in given environments due to the masking of the satellites signals. Consequently, despite the research efforts in this domain and some encouraging results [6], GPS is not yet ready to be chosen and the first positioning technology to be integrated on CIRPAV is LaserGuide.

\section{LaserGuide solution}

LaserGuide [3] is essentially a new form of automatic theodolite which has some similarity to a laser level in that it emits a compound laser beam that rotates in azimuth about a vertical axis through the instrument (currently at $1 \mathrm{~Hz}$ ). The compound beam is formed as a pair of diverging fans, and when incident on a flat vertical surface would generate a "V" pattern.

The essential features of this design of instrument are that the spatial direction to any suitable target ( photocell or glass corner-cube prism, called "beacons") which is within the vertical coverage of the beams, and at any azimuth, may be determined for every scanning cycle of the compound laser beam. This is achieved by use of a single angle encoder within the instrument; as compared with both a vertical and horizontal scale of a conventional theodolite. By computation, knowing the exact geometry of the laser fans, it is possible to determine, from the encoder measurements, complete position and attitude of the LaserGuide instrument in a reference survey frame attached to the beacons. Those beacons are placed in the environment of the site, on perfectly well known survey points.

The beacons which have been developed in CIRC are mainly composed of an electronic photo-cell and a laser transponder which transmit back to the instrument a pulse as soon as it is illuminated by one of the beams. An automatic tracking system allows the beacon to face the moving paver supporting the instrument permanently.

Figure 5 presents the instrument a one of the beacons mounted on a survey tripod.

Given the extreme accuracy of the basic azimuth angle measurements ( 1 to 2 seconds of arc), the final accuracy achieved by the LaserGuide under real operation conditions is impressive. Tests performed on SESSYL test facility at LCPC Nantes centre proved that the accuracy was better than $1 \mathrm{~cm}$ horizontally and $5 \mathrm{~mm}$ vertically, measurements being corrupted by a zero-mean noise (mainly due to the vibrations of the chariot) quite easy to remove with a simple averaging. 

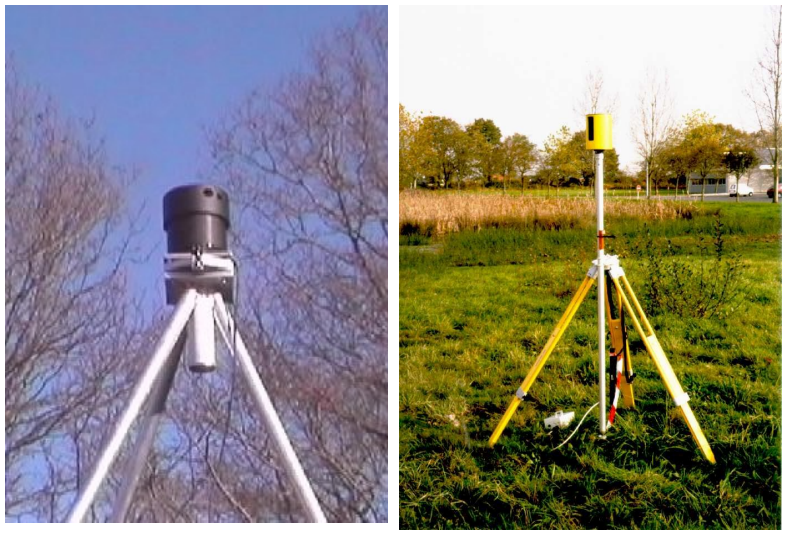

Figure 5: the LaserGuide instrument on top of its trolley and one of the beacons

From an operational point of view, in order to get rid of the screed's vibrations, to make as simple as possible the installation and calibration procedures and to avoid the obstructions problems due to the other machines, a specific trolley has been designed and built to support the LaserGuide instrument. In this respect, CIRPAV can be considered as an advanced levelling system, taking into account for the first time the final result of the process to control, that is to say the spread surface itself.

Figure 6 shows the trolley towed by the paver during the final experimentation.

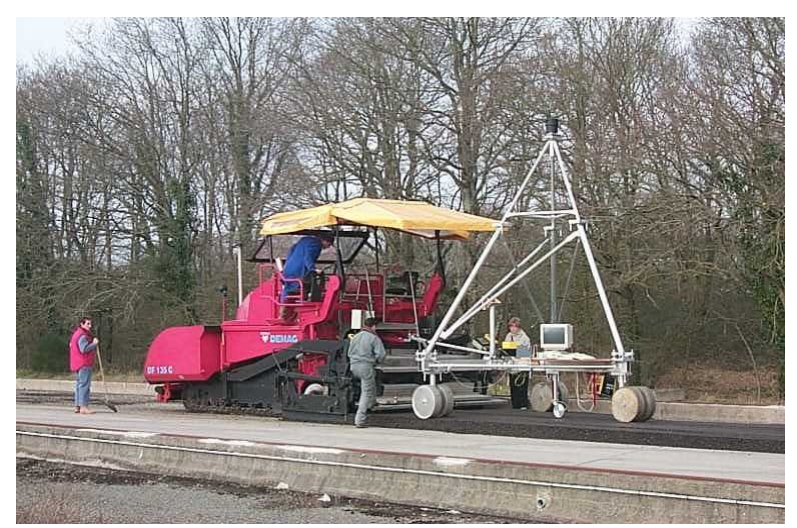

Figure 6: the CIRPAV paver towing the trolley

\subsubsection{On-board sub-system}

The role of the on-board sub-system, embedded on the paver is to:

- automatically control the height and the cross slope of the screed (if the operator chooses the "automatic control" mode),

- manage man-machine interfaces which assist the driver and the screed-man in their work, data,

- process in real time instruction data and position

- memorise work done (3D laid surface).

The CIRPAV MMI follows similar principles to that of CIRCOM, but has more functions. It is particularly dedicated to the driver but displays also all the necessary information to control the screed position from the driver's seat.

Figure 7 shows the screen of the MMI.
Three graphic presentations: work site map, screed view and trajectory display support the driver and screed-man in following the three-dimensional trajectory designed for the machine and its tool as well as providing an overview of the achieved and scheduled work.

The overview map and trajectory display help the machine operators to maintain correct orientation on the work site. The colour map of levelling errors and both graphic and digital displays of screed position give direct visual feedback in manual control mode and provide additional safety checks in the automatic mode.

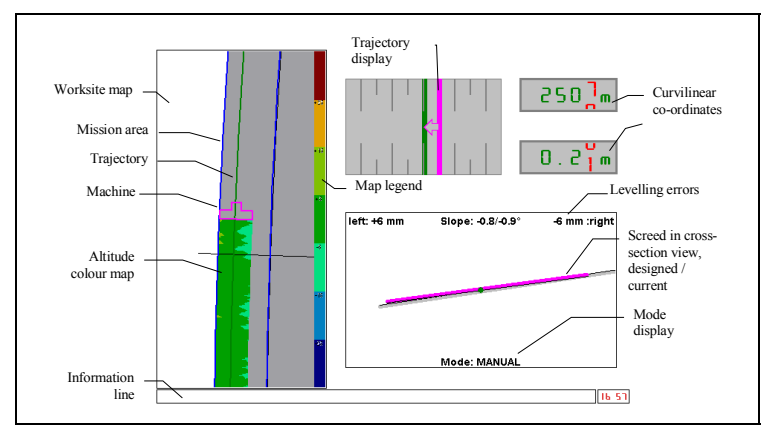

Figure 7: CIRPAV on-board sub-system MMI

The altitude deviations at both extremities of the screed are also repeated on the low-level hydraulic jacks control boxes (such as MOBA-matic for instance), for the screed man.

\section{PROGRESS AND ACHIEVEMENTS}

\subsection{CIRCOM}

\section{Mono-CIRCOM}

The CIRCOM prototype has been developed during the first half of the project in its mono-machine version. Experimentation trials have been organised on the Eurovia A84 motorway work-site in Villedieu-lesPoêles (Normandy, France) at the beginning of September, 1998. These trials were programmed in order to finalise the experimentation (assessment of all the technical functions) and to carry out the siteoriented trials during several days of work necessary (assessment of the operational functions). Numerous remarks collected from the operators have been of great help to design the next generation.

\section{Multi-CIRCOM}

Following strong requirements expressed by the end users at the beginning of the project, the multicompactors functions have been designed and developed between October 1998 and October 1999, as additional work, financially supported by the French Ministry of Transport. To assess the specific multi-compactors features, other field trials, similar to the first ones were organised on another Eurovia work site in Darnieulles (Vosges, France), in early October 1999, involving two real compactors and one simulated one. 
These trials were also successful. Drivers found very satisfying to see their own position compared to the one of their colleague. It helps them in their work especially for minimising the joints between passes, and also of course allow to continue the work started by the other.

\section{CIRCOM pre-industrialisation}

In parallel with multi-CIRCOM related developments, the CIRCOM system (in monocompactor version) has benefited from a preindustrialisation study. This study has allowed the CIRC consortium to enter true commercial negotiations with members of the end-users club. Issuing these negotiations, a first CIRCOM system has been manufactured and delivered beginning of November 1999 to a well-known machine manufacturer : Svedala, his final objective being to integrate as first mount the CIRC devices into his product lines. After preliminarily and successful onsite tests, Svedala demonstrated successfully the product to more than one hundred of his customers in December 1999 and many of them expressed their enthusiasm towards the product.

So, as far as CIRCOM is concerned, the major bases of an effective exploitation has been built during the project. From these bases, a complete productionto-marketing structure is currently being finalised with various European partners and will be ready for the first orders to come at the end of 2000.

\subsection{CIRPAV}

The integration phase was achieved at the end of year 1999, on a DF 135 C Demag finisher, lent by Svedala group to the CIRC consortium. Two MOBAmatic control boxes have also been lent by Moba company to complete the automatic control loops.

The experimentation took place in Nantes during January and February 2000, on the paving test facility of LCPC, with artificial cold material. To determine the position of the paver and the level of the course has been used the LaserGuide prototype, mounted on its trailer, as described above.

During this phase, were carried out the tests necessary to assess the main functions specified by the end-users, with the performances required, in terms of robustness, accuracy, precision, ergonomic, etc. This first phase has been successfully achieved.

The CIRC project officially terminated on the $1^{\text {st }}$ of march, with the presentation of the final results to the European Commission and to several end users.

Concerning CIRPAV, the product will benefit from the CIRCOM exploitation structure mentioned above, but some more developments need to be completed before the prototype is ready to enter the production phase. These works, concerning particularly the positioning sub-system and the automatic control, will be carried out by the concerned partners, in other research frames.

\section{REFERENCES}

[1] F. Peyret, H. Philippe, "Towards Computer Integrated Road Construction", Proc. $9^{\text {th }}$ International Symposium on Automation and Robotics for Construction, Tokyo (1992), 859-868

[2] H. Legentil, Y. Martineau, "A83 : une innovation dans le guidage automatique des finiseurs", Revue Générale des Routes et Aérodromes $n^{\circ} 713$ (1993)

[3] B.J. Gorham, "The LaserGuide system of automatic machine guidance", Proc. $11^{\text {th }}$ International Symposium on Automation and Robotics in Construction, Brighton (1994) 327-332

[4] M. Froumentin, F. Peyret, "An operator aiding system for compactors", Proc. 13 ${ }^{\text {th }}$ International Symposium on Automation and Robotics for Construction, Tokyo (1996) 359-368

[5] M. Froumentin, F. Peyret, Y. Martineau, "Le GPS vient en aide au conducteur du compacteur", Revue Générale des Routes et Aérodromes $n^{\circ} 750$ (1997) 40-47

[6] F. Peyret, D. Bétaille, G. Hintzy, "High-precision application of GPS in the field of real-time equipment positioning", Proc. $14^{\text {th }}$ International Symposium on Automation and Robotics for Construction, Pittsburgh (1997) 2-10

[7] LH. Pampagnin, F. Martinez, F. Peyret and al., "A new GPS-based guiding system for compactors", Proc. $15^{\text {th }}$ International Symposium on Automation and Robotics for Construction, Munich (1998) 153162

[8] L.H. Pampagnin, F. Peyret, G. Garcia, "Architecture of a GPS-based guiding system for road compaction", Proc. IEEE International Conference on Robotics and Automation (ICRA 98), Leuven (1998)

[9] F. Peyret, "The paradigm of computer integrated construction applied to civil-engineering", Proc. $16^{\text {th }}$ International Symposium on Automation and Robotics for Construction, Madrid (1998) 3-10

[10] F. Peyret, "The CIRC project", Proc. $16^{\text {th }}$ International Symposium on Automation and Robotics for Construction, Madrid (1998) 3-10 